\title{
Mitochondrial cardiomyopathy due to m.3243A > G mitochondrial DNA mutation presenting in late adulthood: a case report
} \author{
WL Law33, MS, FRCS

\begin{abstract}
${ }^{1}$ Department of Medicine, Hong Kong Sanatorium \& Hospital, Hong Kong
${ }^{2}$ Department of Pathology, Hong Kong Sanatorium \& Hospital, Hong Kong

${ }^{3}$ Department of Surgery, Hong Kong Sanatorium \& Hospital, Hong Kong
\end{abstract}

Elaine MC Chau ${ }^{1 *}$, MB, BS, FRCP, Edmond SK Ma², MD, FRCP, Annie OO Chan', MD, FRCP, TH Tsoi', MB, BS, FRCP,

Hong Kong Med J 2020;26:240-2

* Corresponding author: echau@hksh.com

https://doi.org/10.12809/hkmj198051

\section{Case report}

Mitochondrial cardiomyopathy usually presents in childhood and is estimated to occur in $20 \%$ to $40 \%$ of children with mitochondrial disease. However, it is a rare cause of cardiomyopathy in adults. We report a case of heart failure due to hypertrophic cardiomyopathy presenting in a 57-year-old female patient with intestinal pseudo-obstruction and bilateral sensorineural deafness. The constellation of diseased organs led to suspicion of underlying mitochondrial disease, subsequently confirmed by genetic mitochondrial DNA (mtDNA) testing that revealed the m.3243 $\mathrm{A}>\mathrm{G}$ pathogenic variant in the MT-TL1 gene.

A 57-year-old female was referred for investigation of recent-onset congestive heart failure with cardiomegaly and bilateral pleural effusions on chest X-ray together with an elevated serum $\mathrm{N}$-terminal pro-brain natriuretic peptide level of $241 \mathrm{pg} / \mathrm{mL}$ (normal $<100 \mathrm{pg} / \mathrm{mL}$ ). She presented with increasing abdominal distension and dyspnoea for 4 months. She had colonic pseudo-obstruction that did not resolve following repeated decompression and was referred for cardiac assessment with reference to fitness for colectomy under general anaesthesia. Apart from gradual hearing loss over the previous 8 years, she had been previously well. She suffered a miscarriage at age 23 years but subsequently gave birth to two apparently healthy daughters at age 32 and 36 years. Her main complaint over the previous year was constipation. There was no history of diabetes, muscle weakness, or visual problems. The older daughter was reported to have a squint since childhood, suggestive of ophthalmoplegia. There was no family history of any hearing, neurological, gastrointestinal or cardiac problems.

Clinically the patient was in distress and pain due to the grossly dilated abdomen from the colonic pseudo-obstruction (Fig 1). Positron emission tomography-computed tomography scan showed dilated bowel (up to $10 \mathrm{~cm}$ in diameter) from the caecum to the anus but no evidence of malignancy.

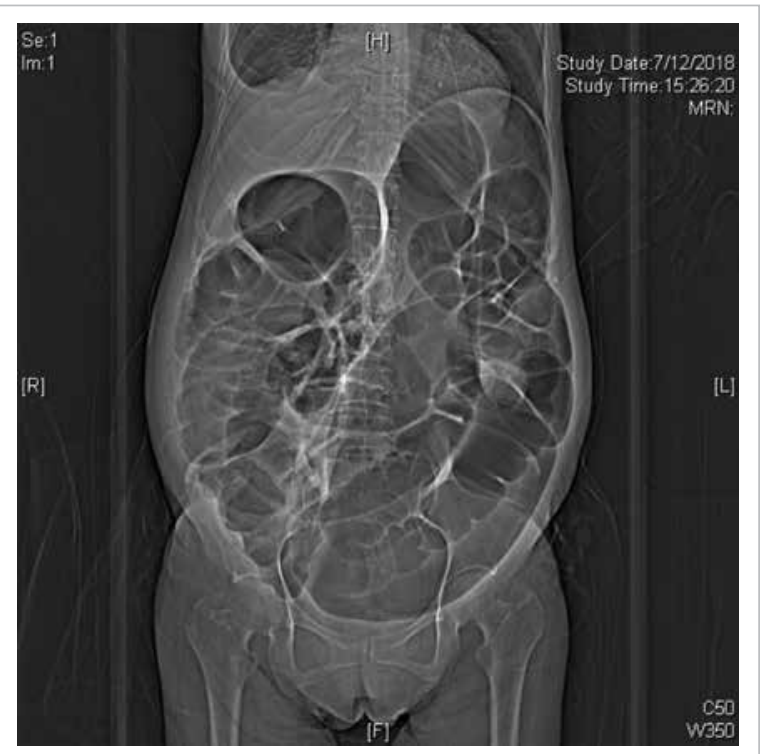

FIG I. Computed tomography abdomen showing grossly dilated large bowel

Electrocardiogram showed sinus rhythm with left ventricular hypertrophy by voltage criteria and strain pattern. Echocardiogram showed left ventricular hypertrophy with mildly impaired left ventricular systolic function (ejection fraction 40\%), diastolic dysfunction and a circumferential pericardial effusion (Fig 2). Computed tomography coronary angiogram was normal. Magnetic resonance imaging (MRI) heart revealed increased $\mathrm{T} 1$ mapping but no late gadolinium enhancement. Screening for amyloidosis was negative. Neurological assessment revealed no evidence of muscle weakness and the creatine kinase level was normal. Computed tomography brain showed dense calcification in bilateral lentiform nuclei and MRI brain was essentially normal.

In view of pending rupture of the dilated colon (up to $10 \mathrm{~cm}$ ), she underwent subtotal colectomy uneventfully. Histopathology of the resected colon 


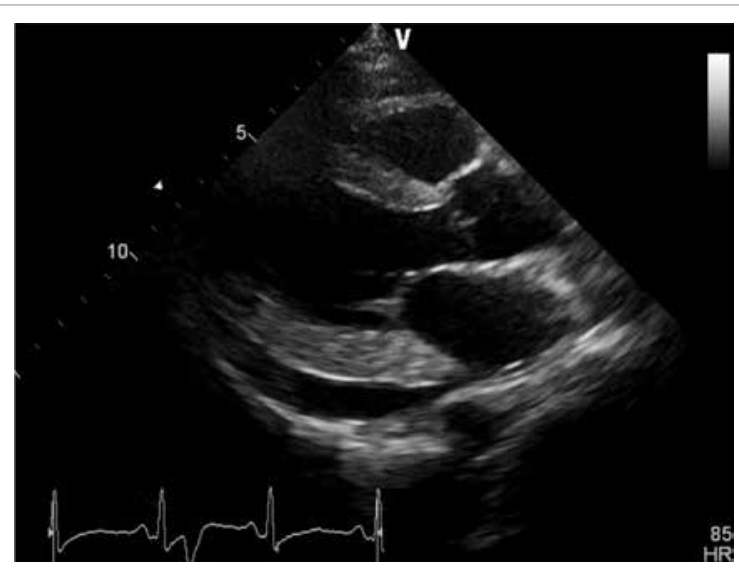

FIG 2. Two dimension-echocardiogram (parasternal long-axis view) showing left ventricular hypertrophy and a moderate amount of pericardial effusion showed no evidence of dysplasia or neoplasia. Blood was sent for mitochondrial disorder testing and showed a m.3243A $>$ G mutation in the MT-TL1 gene, with approximately $10 \%$ heteroplasmy. The resected large bowel was micro-dissected for heteroplasmy determination of $\mathrm{m} .3243 \mathrm{~A}>\mathrm{G}$ by droplet digital polymerase chain reaction. The heteroplasmy levels for smooth muscle, epithelium and connective tissue of the large bowel were $77 \%, 66 \%$ and $46 \%$, respectively.

\section{Discussion}

Mitochondrial DNA disease is a multi-system disorder that affects tissues with high energy demands such as the heart, brain, muscle, and endocrine system. It is caused by mutations in either mtDNA or nuclear DNA genes. Defects in mtDNA can be either point mutations or re-arrangements such as deletions or duplications. The m.3243A $>$ G variant within the MT-TL1 gene (encoding the mitochondrial transfer RNA) is a point mutation and the most common heteroplasmic mtDNA disease genotype. This mutation accounts for approximately $80 \%$ of the MELAS phenotype (mitochondrial myopathy, encephalopathy, lactic acidosis, plus stroke-like episodes) that features mitochondrial encephalomyopathy, lactic acidosis and strokelike episodes, and which this patient did not have. Other phenotypic variations associated with the $\mathrm{m} .3243 \mathrm{~A}>\mathrm{G}$ mutation include maternally inherited diabetes and deafness, ocular (eg, progressive external ophthalmoplegia), gastrointestinal, cardiac, and renal involvement. Most carriers of this mutation are asymptomatic due to mitochondrial heteroplasmy, implying the existence of two or more genomes within the same cell. Usually, the proportion of heteroplasmy needs to exceed a threshold level of $60 \%$ to $90 \%$ for the organ to be clinically affected. The onset and extent of clinical disease are determined by the mtDNA mutation load and threshold. In m.3243A $>\mathrm{G}$ mutation carriers, hypertrophic cardiomyopathy is found in about $18 \%$ of patients. ${ }^{1}$

In a small study of $10 \mathrm{~m} .3243 \mathrm{~A}>\mathrm{G}$ mutation carriers, the skeletal muscle mutation load appeared to correlate with the measures of early cardiac dysfunction on MRI, namely the torsionto-endocardial strain ratio and radial thickening. ${ }^{2}$ Different patterns of cardiac involvement are associated with different mtDNA mutations. Although hypertrophic cardiomyopathy is frequently associated with mt-tRNA (transfer RNA) mutations such as the m.3243A $>\mathrm{G}$ mutation, restrictive cardiomyopathy is associated with the m.1555A $>\mathrm{G}$ mitochondrial ribosomal ribonucleic acid gene mutation. In children with Kearns-Sayre syndrome due to single, large-scale deletions in mtDNA, complete heart block is common. ${ }^{3}$ In our case, the heteroplasmy level within the different structures in the large bowel (46\%-77\%) was uniformly higher than in the blood $(10 \%)$. This was consistent with the clinical presentation of pseudo-obstruction with the large bowel being the major organ involved.

Unfortunately, there is no cure for mitochondrial disease. Device therapy in the form of pacemakers and cardioverter-defibrillators may be required for heart block and ventricular arrhythmias associated with mitochondrial cardiomyopathy. Due to the complexity of mitochondrial disease, it is now recommended that affected patients be assessed using a validated semi-quantitative clinical rating scale, the Newcastle Mitochondrial Disease Adult Scale, to evaluate the different systems involved and to monitor response to treatment. Regular cardiovascular screening is recommended with electrocardiogram and echocardiogram performed at least every 2 years. ${ }^{4}$

Mitochondrial DNA is strictly maternally inherited, with only rare reports of paternal transmission. This may be due to a dilution effect since the sperm contain only 100 copies of mtDNA compared with 100000 copies in the unfertilised egg and, secondly, there is elimination of sperm mtDNA in normal embryos. The m.3243 $\mathrm{A}>\mathrm{G}$ mutation is usually transmitted from mother to offspring with rare reported cases of de novo mutation. ${ }^{5}$ Genetic counselling in mitochondrial disease may be difficult because of heteroplasmy, mitotic segregation, and mitochondrial bottleneck phenomenon. Phenotypic variations associated with $\mathrm{m} .3243 \mathrm{~A}>\mathrm{G}$ are due to a different percentage of mutation heteroplasmy in affected organs. It is known that an asymptomatic mother with a subclinical heteroplasmy level can give birth to an affected child with a higher heteroplasmy 
level. This is explained by the mitochondrial bottleneck theory, whereby redistribution of mtDNA to daughter cells during oocyte production and amplification of the redistributed mtDNA during oocyte development occurs. The unequal mitotic segregation of mtDNA during cell division and the reduction/amplification event leads to a random shift of mtDNA mutational load between generations. This is thought to be responsible for the variable levels of mutation heteroplasmy observed in affected offspring from mothers with pathogenic mtDNA mutations.

\section{Conclusion}

Mitochondrial cardiomyopathy is a rare cause of heart failure presenting in adulthood. Mitochondrial disease should be suspected when other organs or tissues are involved. Genetic testing is a powerful diagnostic tool. Severity and prognosis of mitochondrial disease depend on the concentration of heteroplasmy in the affected organs. Screening and follow-up cardiac assessment are recommended due to the progressive nature of cardiac involvement and risk of heart failure and arrhythmias as a cause of death in patients with mitochondrial mutation. Mitochondrial mutations are maternally inherited but the unique features of mitochondrial inheritance, namely mitotic segregation and mitochondrial bottleneck phenomenon, make predictions of inheritance a challenge. Genetic counselling should be offered to the families of an affected individual.

\section{Author contributions}

Concept or design: All authors.

Acquisition of data: EMC Chau, ESK Ma.

Analysis or interpretation of data: EMC Chau.
Drafting of the manuscript: EMC Chau.

Critical revision for important intellectual content: All authors.

The authors had contributed to the manuscript, approved the final version for publication, and take responsibility for its accuracy and integrity.

\section{Conflict of interest}

The authors have no conflicts of interest to disclose.

\section{Funding/support}

This case report received no specific grant from any funding agency in the public, commercial, or not-for-profit sectors.

\section{Ethics approval}

The patient was treated in accordance with the Declaration of Helsinki. The patient provided informed consent for all procedures.

\section{References}

1. Pankuweit S, Richter A. Mitochondrial disorders with cardiac dysfunction: an under-reported aetiology with phenotypic heterogeneity. Eur Heart J 2015;36:2894-7.

2. Hollingsworth KG, Gorman GS, Trenell MI, et al. Cardiomyopathy is common in patients with the mitochondrial DNA m.3243A $>$ G mutation and correlates with mutation load. Neuromuscul Disord 2012;22:592-6.

3. Bates MG, Bourke JP, Giordano C, d'Amati G, Turnbull DM, Taylor RM. Cardiac involvement in mitochondrial DNA disease: clinical spectrum, diagnosis, and management. Eur Heart J 2012;33:3023-3.

4. Schaefer AM, Phoenix C, Elson JL, McFarland R, Chinnery PF, Turnbull DM. Mitochondrial disease in adults: a scale to monitor progression and treatment. Neurology 2006;66:1932-4.

5. de Laat P, Janssen MC, Alston CL, Taylor RW, Rodenburg RJ, Smeitink JA. Three families with 'de novo' m.3243A>G mutation. BBA Clin 2016;6:19-24. 